\title{
On the distance, reddening and progenitor of V838 Mon
}

\author{
U. Munari ${ }^{1}$, A. Henden ${ }^{2}$, A. Vallenari ${ }^{3}$, H. E. Bond ${ }^{4}$, R. L. M. Corradi ${ }^{5}$, L. Crause ${ }^{6}$, \\ S. Desidera ${ }^{3}$,E. Giro ${ }^{3}$, P. M. Marrese ${ }^{1}$, S. Ragaini ${ }^{3}$, A. Siviero ${ }^{1}$, R. Sordo ${ }^{1}$, \\ S. Starrfield ${ }^{7}$, T. Tomov ${ }^{8}$, S. Villanova ${ }^{9}$, T. Zwitter ${ }^{10}$, and R. M. Wagner ${ }^{11}$ \\ 1 INAF - Osservatorio Astronomico di Padova, Sede di Asiago, 36012 Asiago (VI), Italy \\ e-mail: munari@pd.astro.it \\ 2 Univ. Space Research Assoc./US Naval Observatory, PO Box 1149, Flagstaff AZ 86002-1149, USA \\ 3 INAF - Osservatorio Astronomico di Padova, Vicolo dell'Osservatorio 8, 35122 Padova, Italy \\ 4 Space Telescope Science Institute, 3700 San Martin Drive, Baltimore, MD 21218 USA \\ 5 Isaac Newton Group of Telescopes, Apartado de Correos 321, 38700 Santa Cruz de La Palma, Canarias, Spain \\ 6 South African Astronomical Observatory, PO Box 9, Observatory 7935, South Africa \\ 7 Dept. of Physics and Astronomy, Arizona State Univ., PO Box 871504 Tempe, AZ 85287-1504, USA \\ 8 Centre for Astronomy, Nicolaus Copernicus University, ul. Gagarina 11, 87-100 Torun, Poland \\ 9 Dipartimento di Astronomia, Universitá di Padova, 35122 Padova, Italy \\ ${ }^{10}$ University of Ljubljana, Department of Physics, Jadranska 19, 1000 Ljubljana, Slovenia \\ ${ }^{11}$ Large Binocular Telescope Observatory, Univ. of Arizona, 933 North Cherry Avenue, Tucson, AZ 85721, USA
}

\section{Received 29 July 2004 / Accepted 19 January 2005}

\begin{abstract}
Extensive optical and infrared photometry as well as low and high resolution spectroscopy are used as inputs in deriving robust estimates of the reddening, distance and nature of the progenitor of V838 Mon, the 2002 outbursting event that produced a most spectacular light-echo. The reddening affecting V838 Mon is found to obey the $R_{V}=3.1$ law and amounts to (i) $E_{B-V}=0.86$ from the interstellar NaI and KI lines; (ii) $E_{B-V}=0.88$ from the energy distribution of the B3 $\mathrm{V}$ component; and (iii) $E_{B-V}=0.87$ from the progression of extinction along the line of sight. The adopted $E_{B-V}=0.87 \pm 0.01$ is also the amount required by fitting the progenitor with theoretical isochrones of appropriate metallicity. The distance is estimated from (a) the galactic kinematics of the three components of the interstellar lines; $(b)$ the amount of extinction vs. the HI column density and vs. the dust emission through the whole Galaxy in that direction; from $(c)$ spectrophotometric parallax to the B3 V companion; from $(d)$ comparison of the observed color-magnitude diagram of field stars with 3D stellar population models of the Galaxy; from (e) comparison of theoretical isochrones with the components of the binary system in quiescence and found to be around $10 \mathrm{kpc}$. Pre-outburst optical and IR energy distributions show that the component erupting in 2002 was brighter and hotter than the B3 V companion. The best fit is obtained for a $50000 \mathrm{~K}$ source, 0.5 mag brighter than the B3 V companion. The latter passed unaffected through the outburst, which implies an orbital separation wide enough to avoid mass exchange during the evolution of the binary system, and to allow a safe comparison with theoretical isochrones for single stars. Such a comparison suggests that the progenitor of the outbursting component had an initial mass $\sim 65 M_{\odot}$, that it was approaching the carbon ignition stage in its core at the time it erupted in 2002 and that the age of the V838 Mon binary system is close to 4 million yr. The 2002 event is probably just a shell thermonuclear event in the outer envelope of the star.
\end{abstract}

Key words. stars: evolution - stars: early-type - stars: individual: V838 Mon - stars: winds, outflows ISM: lines and bands - stars: peculiar

\section{Introduction}

V838 Mon rose from obscurity in early January 2002, when it was discovered in outburst by Brown (2002). The unusually cool spectrum and strange light-curve helped to keep attention focused on the object for the next three months, until the discovery in late March by Henden et al. (2002) of a

* Tables 3 and 4 are available only in electronic form at the CDS via anonymous ftp to cdsarc.u-strasbg.fr $(130.79 .128 .5)$ or via http://cdsweb.u-strasbg.fr/cgi-bin/qcat? J/A+A/434/1107 light-echo rapidly developing around V838 Mon. The presence of the first Galactic light echo in $\sim 70$ years fostered a massive, multi-wavelength observing campaign for V838 Mon. A high spatial resolution imaging series of the light-echo expansion and evolution was collected with HST by Bond et al. (2003), recently expanded by new images secured within the Hubble Heritage Program. An account of the spectroscopic, photometric and polarimetric evolution of V838 Mon during the first season of visibility was presented by Munari et al. (2002a). A major observational constraint was the discovery by 
Desidera \& Munari (2002) and Munari et al. (2002b) that V838 Mon is a binary system containing a normal B3V star, implying a young age and $M \geq 7 M_{\odot}$ for its outbursting companion. Over the last two years, V838 Mon has been the topic of numerous refereed papers and conference reports, with a sample given below. IR photometry and spectroscopy was presented and discussed by Banerjee \& Ashok (2002), Crause et al. (2003), Evans et al. (2003) and Rushton et al. (2003), optical polarimetry and spectropolarimetry by Wisniewski et al. (2003a,b) and Desidera et al. (2004), optical photometry by Kimeswenger et al. (2002), Barsukova et al. (2002), Kato (2003) and Goranskii et al. (2004), and optical spectroscopy by Munari et al. (2002a,c), Goranskii et al. (2002), Kolev et al. (2002), Osiwala et al. (2002), Kipper et al. (2004). Modeling of the outburst was presented by Retter \& Marom (2003), Soker \& Tylenda (2003) and Boschi \& Munari (2004), while modeling of the light-echo expansion was performed by Bond et al. (2003), Sugerman (2003) and Tylenda (2004).

Common to many of these papers were the issues of reddening, distance and nature of the progenitor of V838 Mon. However, none of these papers focused specifically on these topics, and limited their discussion to the derivation or adoption of approximate values based on assumed energy distribution of the outbursting component. The aim of the present paper is to attempt an accurate derivation of reddening and distance of V838 Mon by careful comparison of different techniques and approaches that do not involve the outbursting component, as well as to derive by comparison with theoretical models the nature and evolutionary state of V838 Mon prior to the onset of the outburst.

Optical and IR photometry used in this paper was obtained with CCD-equipped USNO Flagstaff Station (NOFS) telescopes. The $U B V R_{\mathrm{C}} I_{\mathrm{C}}$ data were obtained with the $1.0 \mathrm{~m}$ telescope, the $J H K^{\prime} L^{\prime}$ with the $1.55 \mathrm{~m}$ telescope. The optical photometry is strictly tied to the Landolt $(1983,1992)$ system of equatorial standards, while the infrared photometry uses standard $J H K^{\prime}$ filters and differential measures with respect to local 2MASS stars. The low resolution optical spectrophotometry of V838 Mon was obtained with the AFOSC+CCD imager+spectrograph of the $1.82 \mathrm{~m}$ telescope operated in Asiago by the Astronomical Observatory of Padova. At the same telescope we secured high resolution spectra with the Echelle + CCD spectrograph. Other high resolution spectra of V838 Mon were obtained with the Coude spectrograph+CCD of the Rozhen $2 \mathrm{~m}$ telescope, with GIRAFFE at the $1.93 \mathrm{~m}$ SAAO telescope, with SARG at the $3.5 \mathrm{~m}$ TNG telescope, and with FEROS at the $2.2 \mathrm{~m}$ ESO telescope in La Silla. Further details will be provided below where necessary.

Figure 1 presents a review of the optical and IR photometric evolution of V838 Mon, updated to the end of the third season of visibility in May 2004. With the exception of some earlytime datapoints, the light-curves are entirely based on data from the USNO telescopes and therefore they are highly consistent and free from systematic effects of color transformation between different local photometric systems that would badly affect observations from different observatories of an object with such extreme colors as V838 Mon.

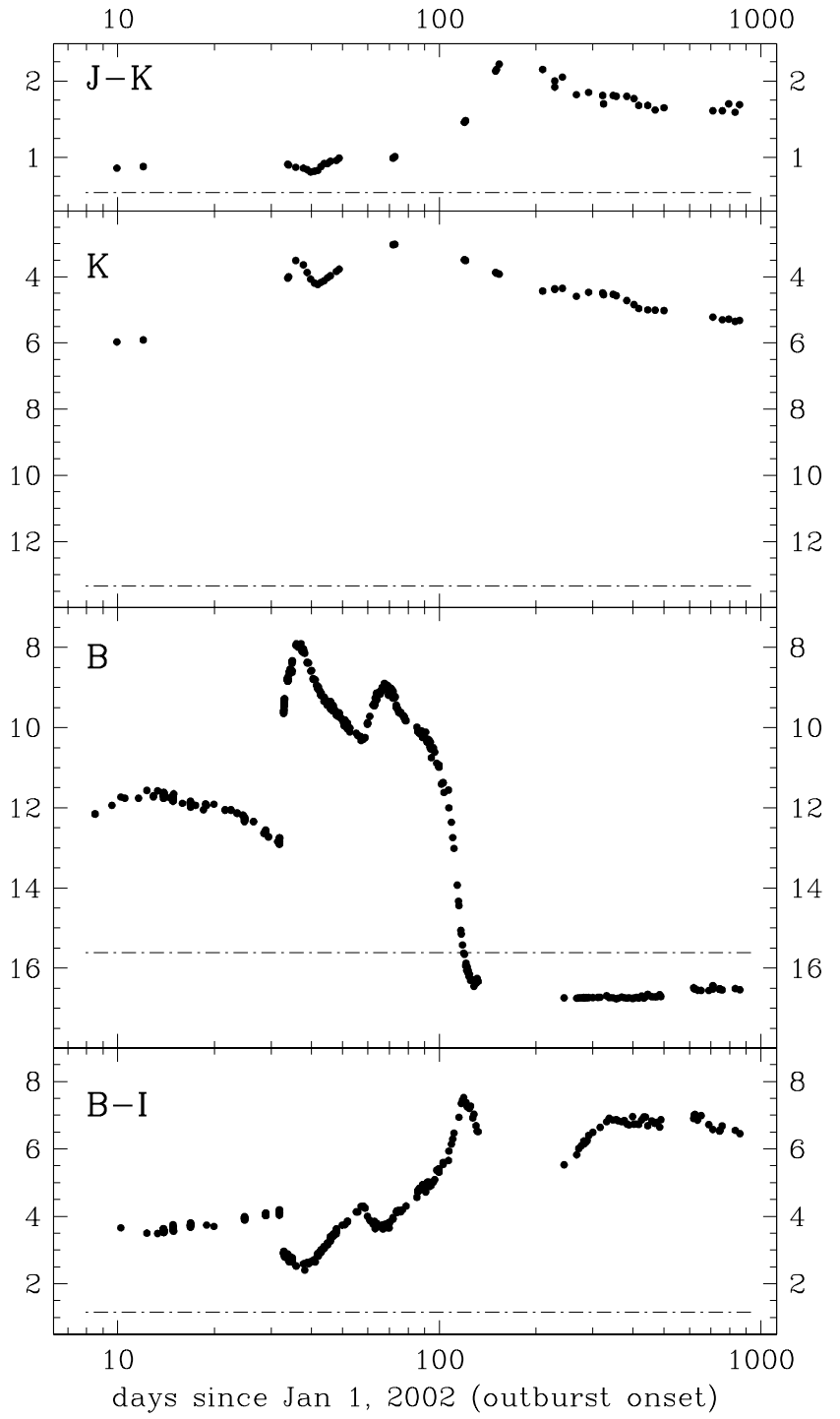

Fig. 1. Optical and IR light-curves of the outburst of V838 Mon as recorded with USNO $1.0 \mathrm{~m}$ and $1.55 \mathrm{~m}$ telescopes; some early-time observations come from other sources as identified in Munari et al. (2002b). The outburst onset is around Jan. 1, 2002, and the first and last observations in the picture are for the dates Jan. 9, 2002 and May 11, 2004, respectively. The gaps in the optical light-curves correspond to seasonal invisibility. The dotted-dashed lines give the values for the quiescence prior to the outburst.

\section{Evidence of patchy extinction}

V838 Mon lies close to galactic equator $\left(b=+1^{\circ}\right)$ and in the anti-center quadrant $\left(l=218^{\circ}\right)$. In spite the line of sight crosses the Orion, Perseus and Outer spiral arms, inspection of the Palomar plates, of the 2MASS, IRAS, UKST-H $\alpha$ maps and the Neckel \& Klare (1980) extinction charts do support an apparently smooth star counts distribution for several degrees around V838 Mon position. Therefore in principle, open clusters and field stars with accurate photometry and spectroscopic classification can be used to search for a relationship between reddening and distance in the direction of V838 Mon.

Within $5^{\circ}$ from V838 Mon there are 13 open clusters in the catalog of Lyngå (1987). They are listed in Table 1. According 
Table 1. Open clusters within $5^{\circ}$ of V838 Mon from the Lynga (1987) catalog. $\theta$ is the angular distance in degrees from V838 Mon. Reddening and distances are taken from the Dias et al. (2002) compilation.

\begin{tabular}{lcccccc}
\hline cluster & $\begin{array}{c}\text { gal. long. } \\
\left({ }^{\circ}\right)\end{array}$ & $\begin{array}{c}\text { gal. lat. } \\
\left({ }^{\circ}\right)\end{array}$ & $\begin{array}{c}\theta \\
\left({ }^{\circ}\right)\end{array}$ & $\begin{array}{c}\text { dist. } \\
(\mathrm{kpc})\end{array}$ & $\begin{array}{c}\text { log age } \\
(\mathrm{yr})\end{array}$ & $E_{B-V}$ \\
& & & & & & \\
Bochum 3 & 218.79 & +00.35 & 1.21 & 1.76 & 7.890 & 0.240 \\
NGC 2311 & 217.76 & -00.69 & 1.75 & 2.29 & 8.600 & 0.330 \\
NGC 2338 & 219.88 & +01.01 & 2.09 & & & \\
Haffner 3 & 219.82 & -00.01 & 2.29 & & & \\
Berkeley 34 & 214.18 & +01.88 & 3.71 & & & \\
NGC 2309 & 219.84 & -02.25 & 3.88 & 2.51 & 8.400 & 0.350 \\
NGC 2306 & 219.69 & -02.60 & 4.12 & & & \\
NGC 2286 & 215.31 & -02.27 & 4.16 & 2.90 & 8.883 & 0.170 \\
Berkeley 77 & 219.36 & +05.17 & 4.40 & & & \\
NGC 2302 & 219.30 & -03.12 & 4.43 & 1.18 & 7.847 & 0.207 \\
NGC 2323 & 221.67 & -01.33 & 4.55 & 0.93 & 8.096 & 0.213 \\
NGC 2324 & 213.45 & +03.30 & 4.89 & 3.81 & 8.630 & 0.127 \\
Berkeley 37 & 217.23 & +05.93 & 4.92 & & & \\
\hline
\end{tabular}

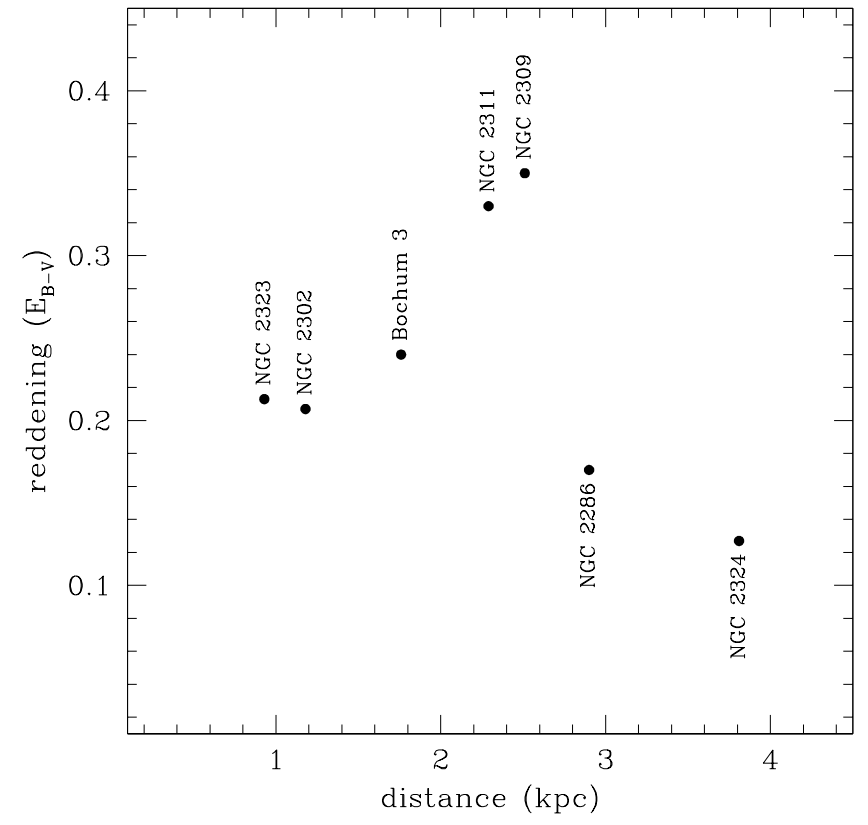

Fig. 2. Extinction as function of distance for open clusters within $5^{\circ}$ from V838 Mon.

to the compilation of Dias et al. (2002), only half of them have published determinations of distance and reddening. The placing of these 7 clusters on a distance-reddening diagram (cf. Fig. 2) shows a large scatter and basically no obvious trend over such angular distances.

The best source of homogeneous spectroscopic classification of field stars is the Michigan Project, which is a continuing program at the University of Michigan where all HD stars are being reclassified on the MK system, starting at the south Galactic pole, and ending at the north Galactic pole. Volume 5 (Houk \& Swift 1999) covers the declination strip $-12^{\circ} \leq \delta \leq$ $5^{\circ}$, thus including the region around V838 Mon.

We selected all HD stars within a radius of $3^{\circ}$ from V838 Mon and retrieved their $B_{\mathrm{T}}$ and $V_{\mathrm{T}}$ magnitudes from the Tycho-2 catalog (Høg et al. 2000). The latter were transformed into corresponding Johnson $B_{\mathrm{J}}$ and $V_{\mathrm{J}}$ following Bessell (2000)

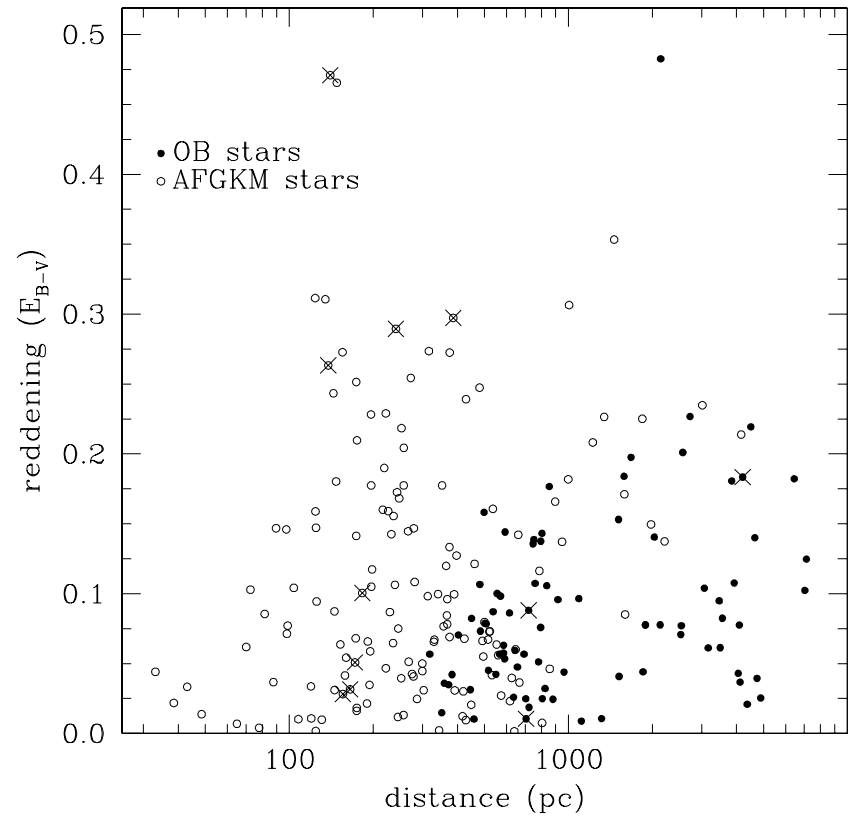

Fig. 3. The HD stars closer than $3^{\circ}$ from V838 Mon are plotted. The crosses mark those within $0.6^{\circ}$ from V838 Mon. Only HD stars classified by the Michigan Project and with Tycho- $2 B_{\mathrm{T}}, V_{\mathrm{T}}$ data are considered.

transformations. The reddening of each star was than derived by comparison with Fitzgerald (1970) intrinsic colors, and the distance computed using the absolute magnitudes of the Michigan Project scale (from N. Houk web page ${ }^{1}$ ).

Figure 3 plots the distribution of HD field stars in a distance-reddening diagram. No well defined progression of the reddening with distance is evident in the figure. The amount of scatter on the diagram is much larger than justifiable in terms of measurement and classification errors, and it clearly confirms the evidence from open clusters that the reddening is very patchy over the sky area centered on V838 Mon in spite of the smooth star counts distribution over the area. Even restricting to the eleven HD stars closer than $0.6^{\circ}$ (marked by crosses in Fig. 3) does not help to define a well-behaved relation between distance and reddening. This indicates that the reddening coherence area around V838 Mon has a significantly smaller radius.

\section{The field stars close to V838 Mon}

Noting the patchy reddening structure over degree-sized areas, we have instead focused on a much smaller angular scale in looking for a coherent relation between distance and reddening along the line of sight to V838 Mon. Deep $U B V R_{\mathrm{C}} I_{\mathrm{C}}$ photometry of field stars within a $11 \times 11$ arcmin field centered on V838 Mon has been obtained with the NOFS $1.0 \mathrm{~m}$ telescope. The observed color-magnitude diagrams of these field stars have then been compared to and fitted with synthetic diagrams

\footnotetext{
1 http://www.astro. lsa.umich.edu/users/hdproj/ mosaicinfo/absmag.html
} 


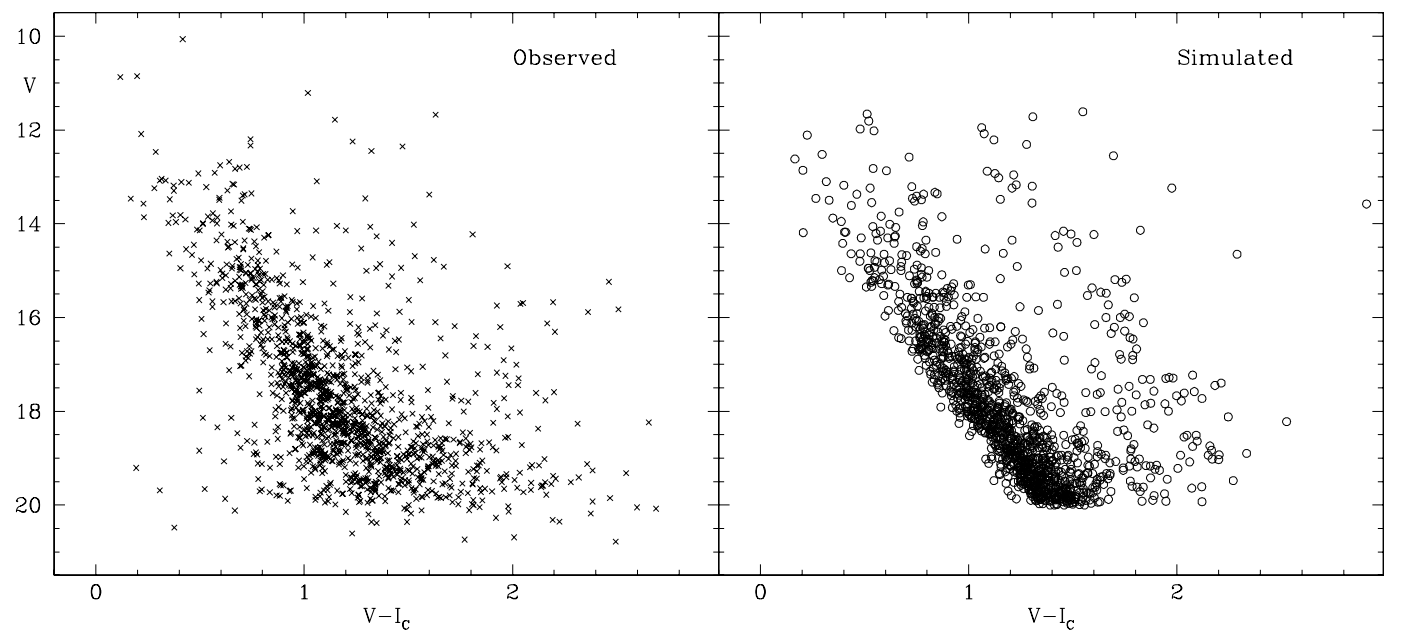

Fig. 4. The left panel plots on a $V, V-I$ diagram the stars within a $11 \times 11$ arcmin field centered on V838 Mon as observed with the NOFS $1.0 \mathrm{~m}$ telescope. The right panel represents the fit to the observed distribution using the Padova Galaxy Model, normalized to the same number of stars (1358).

from stellar population models of our Galaxy. The photometric data of the field stars are electronically available ${ }^{2}$.

V838 Mon lies close to the galactic equator and in the general anti-center galactic direction, where stellar counts are largely dominated by the disk population. Bertelli et al. (1995) showed that the slope of the main sequence of the disk population in color-magnitude diagrams is mainly governed by the extinction along the line of sight. At any $V$ magnitude the bluest stars on the main sequence can be interpreted as the envelope of the main sequence turnoffs of the population having absolute magnitude $M_{\text {tur }}$, shifted toward fainter magnitudes and redder colors by the increasing distance and corresponding extinction. Starting from an initial guess, the amount of extinction at increasing distances is adjusted (via $\chi^{2}$ test on the color distribution as function of magnitude) until a satisfactory agreement between the main sequence blue edge location in the observations and in the model is reached. The distribution of observational errors is evaluated and taken into account in the process.

The Galaxy model that we used is based on the code described by Bertelli et al. (1995) and recently revised by Vallenari et al. (2000, 2004). The stars are generated according to the evolutionary model of the Galaxy, and then are distributed along the line of sight following the spatial model of the Galaxy. All stellar populations are taken into account. The generation of the synthetic population makes use of the set of the stellar tracks by Girardi et al. (2000). A double exponential mass distribution inside the thin disk is adopted having a scale length of $2500 \mathrm{pc}$ and a scale height varying with the age of the population, ending at $350 \mathrm{pc}$ for the oldest component. A thick disk component is included with a scale height of $800 \mathrm{pc}$. A stochastic age metallicity relation for the disk is assumed, with $\mathrm{Z}$ going from 0.008 to 0.03 .

We performed the analysis in both $V, B-V$ and $V, V-$ $I$ planes, with similar results. Figure 4 presents the distribution of observed and synthetic stars in the $V, V-I$ diagram, and Fig. 5 gives the resulting distribution of the extinction $\left(A_{\mathrm{V}}\right)$

\footnotetext{
${ }^{2}$ At http://ulisse.pd.astro.it/V838_Mon/
}

along the line of sight. We experimented with different star formation histories in both the thin and thick galactic disks, but $A_{\mathrm{V}}=f(d)$ never changed by more than 0.1 mag at any point along the line of sight. The values reported in Table 4 and adopted here are those providing the best results in the $\chi^{2}$ tests. The error bars in Fig. 5 include both the effect of observational errors and incompleteness as well as different star formation histories in the galactic disk over the last 10 Gyr. The magnitude of turnoff stars beyond $6 \mathrm{kpc}$ is at $V \geq 19$, where observational errors become significant and completeness begins to be affected. Therefore, the results in Table 4 and Fig. 5 are less accurate beyond $6 \mathrm{kpc}$. To increase the accuracy at larger distances it is advisable that much deeper photometry is obtained in future observations with $8 \mathrm{~m}$ class telescopes. Comparing the HR diagrams of field stars in different colors it is clear that a standard $R_{V}=A_{\mathrm{V}} / E_{B-V}=3.1$ extinction law applies.

In Fig. 5 comparison is made with other $A_{\mathrm{V}}=f(d)$ already presented in literature. Mendez \& van Altena (1998) make use of the large-scale properties of the dust layer in the Galaxy to derive the absorption in the plane of the Galaxy. Their model indicates that a differential optical absorption of $0.5 \mathrm{mag} \mathrm{kpc}^{-1}$ is adequate to reproduce the available reddening maps in the range $2 \leq r \leq 6 \mathrm{kpc}$. The internal uncertainties reported by Mendez \& van Altena are of the order of $E_{B-V}=0.25$, or $A_{V}=0.77 \mathrm{mag}$ for a standard $A_{V}=3.1 \times E_{B-V}$. Schlegel et al. (1998), using the COBE-DIRBE 100 and $240 \mu \mathrm{m}$ all sky survey, derived a map of dust emissivity, which was converted into a map proportional to dust column density and, finally, to a map of the total amount of reddening through any direction in the Galaxy. These maps are expected to suffer in accuracy close to the galactic plane, at $|b|<5^{\circ}$. Drimmel et al. (2003) recently presented a dimensional model of the dust distribution based on COBE-DIRBE infrared data which aims to be useful closer to the Galactic plane, since it accounts for the presence of spiral arms. As stated by Drimmel et al., regions having anomalous emission due to warm dust (like Orion or the Galactic center) are not well described by their model. Neckel \& Klare (1980) extinction maps are based on observed $B-V$ colors of stars 


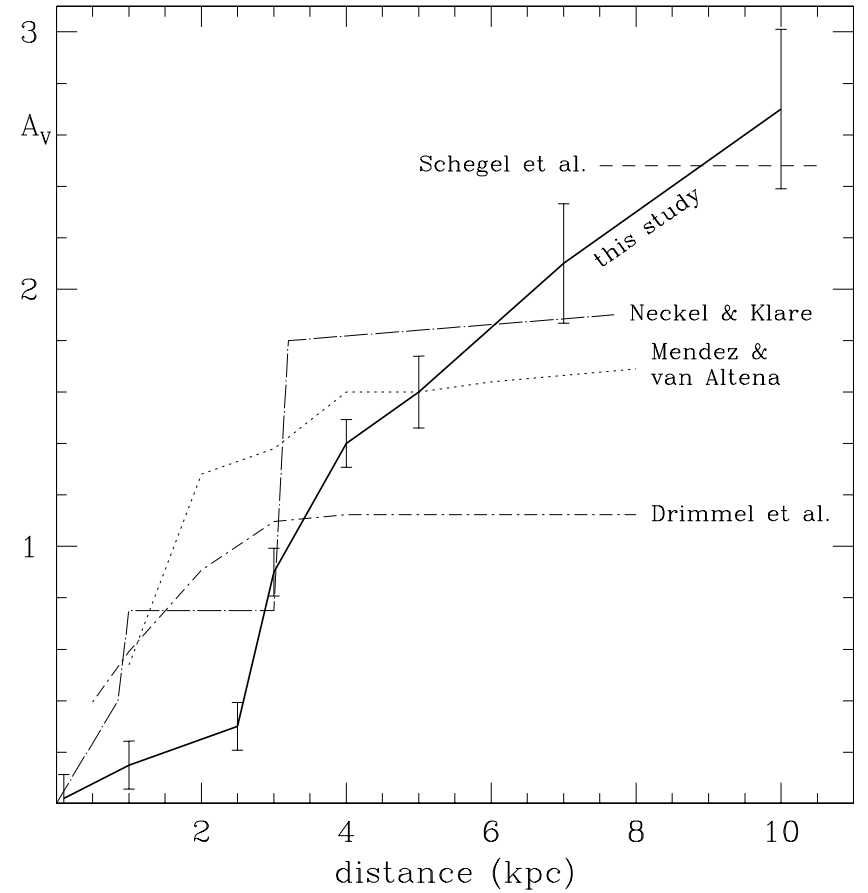

Fig. 5. Extinction along the line of sight to V838 Mon (thick and solid line) as implied by the galactic synthetic population fit in Fig. 4 to the observed color-magnitude diagram of field stars. The dotted line represents the Mendez \& van Altena (1998) model for the dust in the Galaxy, the short dashed-dotted line is the Drimmel et al. (2003) model, while the dashed lines give the total galactic extinction along the line of sight according to the Schlegel et al. (1998) COBE maps. The long dashed-dotted line is the extinction behavior according to the Neckel \& Klare (1980) maps (poorly populated at $>3 \mathrm{kpc}$ ).

of known spectral type and luminosity class so that a spectrophotometric parallax can be estimated. Neckel \& Klare data are only tentative at distances larger than $3 \mathrm{kpc}$ given the paucity of data.

\section{The $\mathrm{Nal}$ and $\mathrm{KI}$ interstellar lines}

The interstellar lines are a powerful mean for deriving the reddening along the line of sight. Munari \& Zwitter (1997) have calibrated accurate relations between reddening and equivalent width of $\mathrm{NaI}$ and $\mathrm{KI}$ interstellar lines that we apply to V838 Mon in this section.

We have secured a number of high resolution spectra during the outburst of V838 Mon which are suitable to reveal and measure the interstellar NaI and KI lines. They are summarized in Table 3 (available only in electronic form at the CDS). The general appearance of interstellar lines in V838 Mon spectra is illustrated in Fig. 6. There are three main components contributing to the profiles of interstellar lines. They are seen well separated in the KI line observed at a resolving power 48000 with FEROS at the $2.2 \mathrm{~m}$ at ESO. The two components at +29 and $+42 \mathrm{~km} \mathrm{~s}^{-1}$ are blended at the lower resolution of the other instruments, particularly in the case of the stronger NaI lines. For this reason these lower-resolution spectra show a narrow red component, at $+65.1 \mathrm{~km} \mathrm{~s}^{-1}$ radial velocity, and a broader blue component, at $+37.7 \mathrm{~km} \mathrm{~s}^{-1}$ radial velocity. It is worth

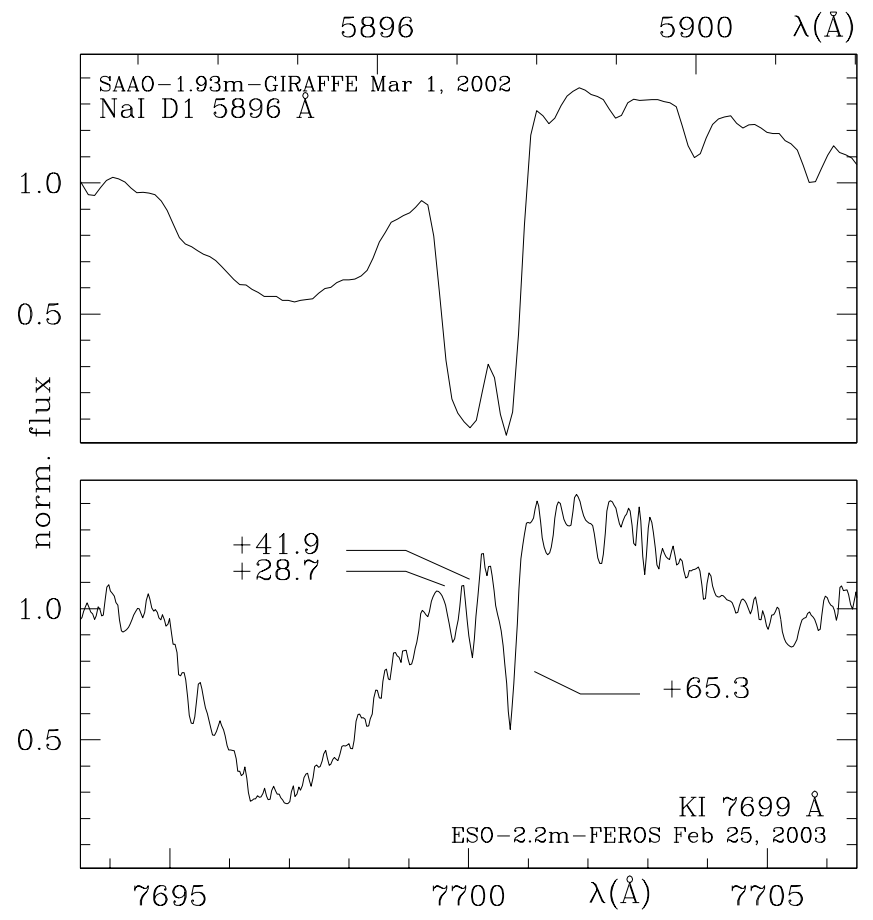

Fig. 6. Interstellar lines in the high resolution optical spectra of V838 Mon. The upper panel shows the profile of the NaI D1 line superimposed on the broad P-Cyg stellar line. The profile is composed of a narrow red component and a blue unresolved blend. The blue blend is resolved in a higher resolution FEROS observations of the KI $7699 \AA$ Al line of V838 Mon on the lower panel.

noting that the average velocity of the two blue KI components $\left(\right.$ at +29 and $\left.+42 \mathrm{~km} \mathrm{~s}^{-1}\right)$ in the FEROS spectrum, weighted according to their equivalent widths, matches exactly the velocity of the blended blue component $\left(+37.7 \mathrm{~km} \mathrm{~s}^{-1}\right)$ in the lower resolution spectra of both $\mathrm{NaI}$ and $\mathrm{KI}$ lines.

The results of the measurement of heliocentric wavelength, equivalent width and FWHM of the NaI and KI lines of spectra listed in Table 3 are given in Table 4 (available only in electronic form at the CDS), separately for the blue and the red components. Their mean values and standard deviation are given in Table 5. Extensive tests on the spectra suggests that the minimal apparent changes with time traceable in Table 4 are not the result of intrinsic variability of the interstellar lines themselves. Instead, they arise from the difficulty (arbitrary) in tracing the level of the underlying continuum in the continuously changing shape of the underlying broad stellar P-Cyg profiles (cf. Fig. 6), as well as the large assortment of spectrographs - each with its own PSF - contributing to the monitoring of the interstellar lines.

The time behavior of heliocentric radial velocity, equivalent width and FWHM is plotted in Fig. 7. It clearly indicates that since earliest outburst phases these values have remained constant, being unaffected by the large changes in the mass loss (amount and velocity) of V838 Mon during its active state. This indicates that the ejected material had not reached and swept away any pre-existing circumstellar material giving rise to $\mathrm{NaI}$ and $\mathrm{KI}$ absorptions, which is consistent with the absence of X-ray detection in Chandra observation 


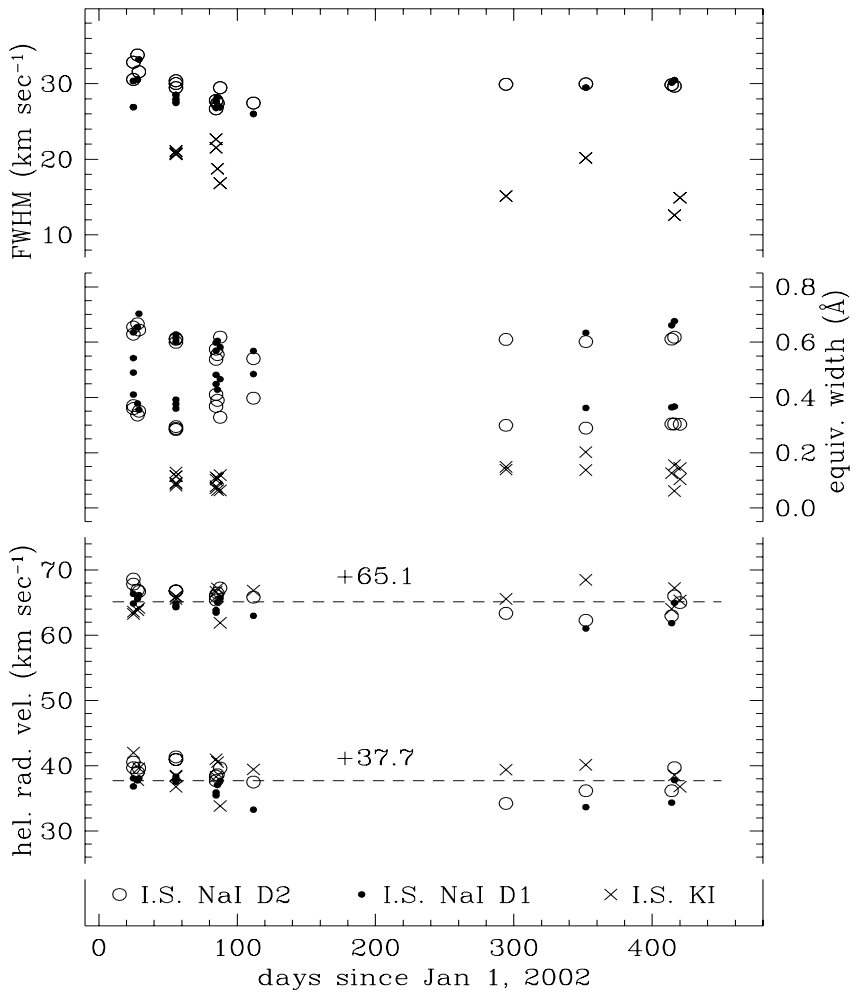

Fig. 7. Heliocentric radial velocities, equivalent widths and full width at half maximum from Table 4 of the interstellar lines as function of time since the onset of the outburst of V838 Mon around Jan. 1, 2002. Their constancy suggests that the violent eruption has not interfered with possible pre-existing circumstellar material.

of V838 Mon by Orio et al. (2003) and the central dust-free region described by Tylenda (2004). All three components of the interstellar lines seem truly interstellar and not circumstellar because ( $a$ ) ground-based and HST images of the light-echo (Munari et al. 2002c; Bond et al. 2003, and a recent Hubble Heritage Program image) show a hole surrounding the central star; $(b)$ the reddening from interstellar lines (cf. next section) matches that from field stars, and above all because; $(c)$ the radial velocities of the three components of the interstellar lines $\left(65,42\right.$ and $\left.29 \mathrm{~km} \mathrm{~s}^{-1}\right)$ precisely correspond to those of the HI complexes $\left(68,43\right.$ and $\left.27 \mathrm{~km} \mathrm{~s}^{-1}\right)$ observed along the line of sight to V838 Mon in the Leiden/Dwingeloo HI Survey (Hartmann \& Burton 1997).

\subsection{Reddening from interstellar lines}

From the wavelength of maximum polarization, Desidera et al. (2004) have demonstrated how the reddening affecting V838 Mon follows the normal $R_{\mathrm{V}}=A_{\mathrm{V}} / E_{B-V}=3.1$ extinction law. The same result follows from the analysis of $U B V R I$ photometry of field stars in Sect. 3. We can therefore safely apply to interstellar lines the Munari \& Zwitter (1997) calibration for the average interstellar medium to the equivalent width of $\mathrm{NaI}$ and $\mathrm{KI}$ lines in Table 5. The resulting reddening is $E_{B-V}=0.86$ from $\mathrm{KI}$ and $E_{B-V}=0.85$ from NaI. The results are in excellent agreement between the two sets of lines. We adopt $E_{B-V}=0.86$ as the reddening indicated by the

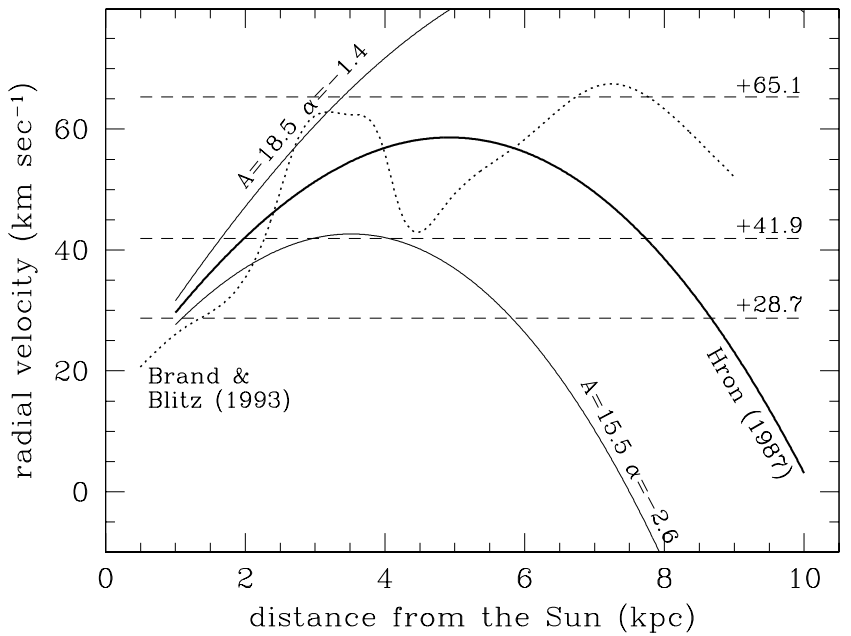

Fig. 8. The radial velocities of the three components of $\mathrm{NaI}$ and $\mathrm{KI}$ interstellar lines $\left(+28.7,+41.9\right.$ and $\left.65.1 \mathrm{~km} \mathrm{~s}^{-1}\right)$ are over-plotted on the velocity field from Brand \& Blitz (1993) and on the rotation curve of the Galaxy of Hron (1987). For the latter two additional curves are plotted, corresponding to the extremes of the allowed ranges for $A$ and $\alpha$ parameters.

interstellar lines, giving higher weight to the results from KI lines which are still far from saturation compared to the nearly saturated NaI lines.

\subsection{Distance from radial velocity of interstellar lines and the galactic rotation}

Some constraints on the distance to V838 Mon can be derived by combining the observed radial velocity of interstellar material and the rotation curve of the Galaxy.

The three principal components of the interstellar absorption lines have heliocentric radial velocities of $+65.1,+41.9$ and $+28.7 \mathrm{~km} \mathrm{~s}^{-1}$. In Fig. 8 they are over-plotted onto the rotation curve of the Galaxy from Hron (1987) and onto the velocity field in the direction of V838 Mon as derived by Brand \& Blitz (1993).

The velocity of the two components at +41.9 and $+28.7 \mathrm{~km} \mathrm{~s}^{-1}$ is compatible with a broad range of distances, while the component at $+65.1 \mathrm{~km} \mathrm{~s}^{-1}$ supports a distance to the absorbing medium not shorter than $3 \mathrm{kpc}$, with a corresponding larger distance for V838 Mon itself. Similar lower limits to the distance to V838 Mon were derived by Wisniewski et al. (2003a) and Kipper et al. (2004) from similar kinematic arguments based on the observed velocity of interstellar lines.

\section{The B3V companion}

Munari et al. (2002c) commented on the fact that the $U B V R_{\mathrm{C}} I_{\mathrm{C}}$ light-curves of V838 Mon started to exhibit a puzzling bluing in $U-B 90$ days and in $B-V 115$ days after the outburst onset, while $V-I$ was rising to very red values, approaching those of brown dwarfs. The reason became apparent when Desidera \& Munari (2002) discovered on spectra at day 274 that the contribution from the very cool outbursting 
component was less in the blue part of the spectrum, revealing the presence of a fainter and hotter companion. V838 Mon was thus shown to be a binary system, and the hot companion was classified by Munari et al. (2002b) on spectra for day 300 as a B3 V star. The presence of the hot companion was confirmed on higher resolution spectra by Wagner \& Starrfield (2002). The composite nature of optical spectra of V838 Mon during this phase is illustrated in the top panel of Fig. 9. Later on there was an increase in the contribution of the outbursting component at shorter wavelengths, as illustrated in the bottom panel of Fig. 9.

The average color of V838 Mon at the time of the naked visibility of the B3 V companion was $B-V=+0.68$ (cf. Fig. 1 and Munari et al. 2002b). Comparing with the intrinsic colors of a B3 V from Fitzgerald (1970) it results in $E_{B-V}=0.88$, in excellent agreement with the results from interstellar $\mathrm{NaI}$ and KI lines.

The metallicity in the galactic disk at the galactocentric distance of $\mathrm{V} 838$ Mon is $[\mathrm{Fe} / \mathrm{H}]=-0.7$, about half dex lower than in the solar neighborhood (e.g. Friel et al. 2002; Hou et al. 2003). The effect on the $B-V$ color of the B3 V component in V838 Mon of such a reduction in metallicity is a very minor one, being on the Rayleigh-Jeans tail of the energy distribution of a hot star. Integrating the transmission profiles of the Landolt's $B$ and $V$ bands (to which our photometry is tied) to the 2500-10500 ̊ synthetic Kurucz spectral library of Munari et al. (2005), the net effect is just $0.007 \mathrm{mag}$.

The average $V$ magnitude of V838 Mon at the time the $V$ band was dominated by the radiation from the B $3 \mathrm{~V}$ component is $V=16.05 \pm 0.05$. Coupled with the $E_{B-V}=0.87 \pm 0.01$ and an absolute magnitude $M_{V}=-1.70 \pm 0.05$ for a B3 V star from Houk (2004), it implies a distance of $10 \mathrm{kpc}$ to V838 Mon.

\section{Combined results}

\subsection{Reddening}

Two assumption-independent methods, the interstellar atomic absorption lines and the colors of the $\mathrm{B} 3 \mathrm{~V}$ companion, provide consistent results on the reddening affecting V838 Mon: $E_{B-V}=0.86$ and $E_{B-V}=0.88$, respectively. There is no differential extinction between the outbursting star and the B3V companion.

The same amount of reddening is derived from the modeling of the HR diagram of field stars close to V838 Mon performed in Sect. 3. In fact, entering Table 2 or Fig. 5 with the distance $(10 \mathrm{kpc})$ to V838 Mon from the spectro-photometric parallax to the B3V companion, $E_{B-V}=0.87$ is obtained.

We therefore conclude that the extinction toward V838 Mon follows the standard $R_{\mathrm{V}}=A_{\mathrm{V}} / E_{B-V}=3.1$ law and the reddening amounts to $E_{B-V}=0.87 \pm 0.01$.

\subsection{Distance}

There are several and independent arguments and evidences that support a long distance scale to V838 Mon, of the order of $10 \mathrm{kpc}$.

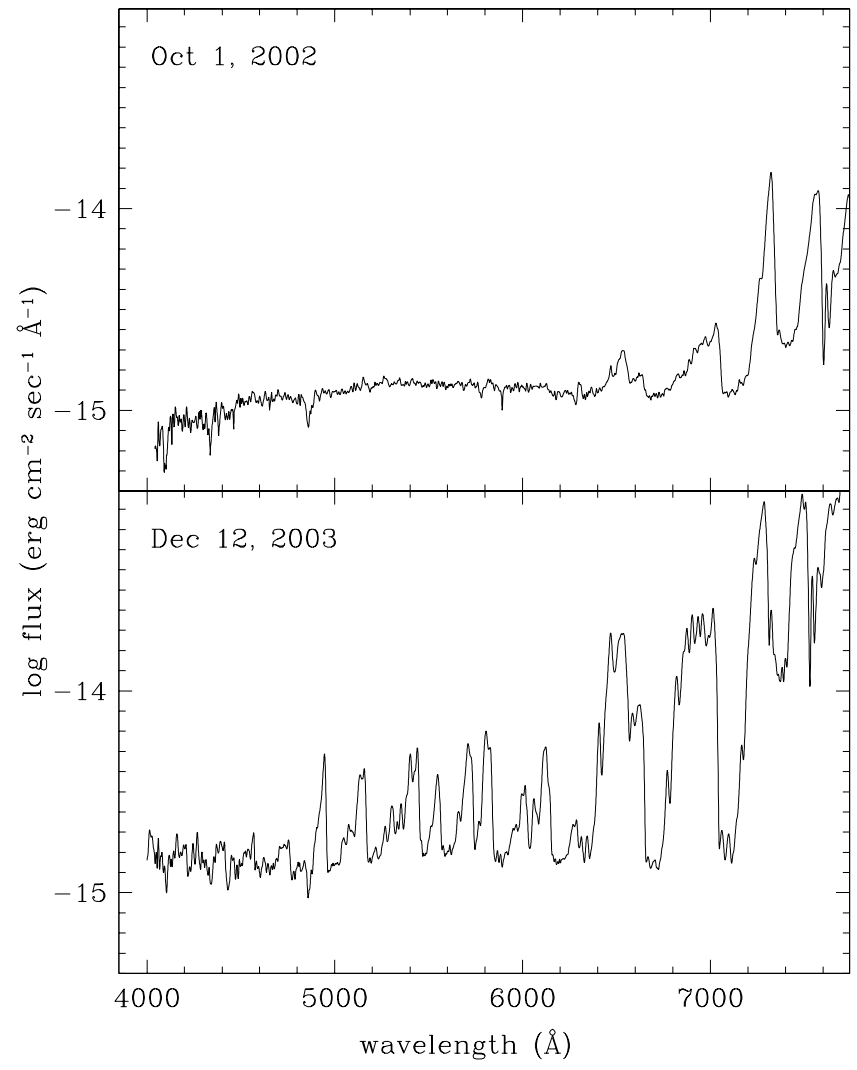

Fig. 9. Comparison between Asiago AFOSC spectra of V838 Mon taken in October 2002 and December 2003. At the earlier date the outbursting component is only visible toward the red, revealing the presence of a B $3 \mathrm{~V}$ companion whose emission dominates the $U, B$ and $V$ bands at that time. The later spectrum illustrates how the emission of the outbursting component has retraced back to shorter wavelengths during the 2003/04 visibility season, a change already described in its early development by Wagner et al. (2003).

Table 2. Absorption along the line of sight to V838 Mon according to the analysis of the color magnitude diagram of the field stars presented in Figs. 4 and 5.

\begin{tabular}{ccccccccc}
\hline \hline Dist (kpc) & 0.1 & 1.0 & 2.5 & 3.0 & 4.0 & 5.0 & 7.0 & 10.0 \\
$A_{\mathrm{V}}$ & 0.01 & 0.15 & 0.3 & 0.7 & 1.4 & 1.6 & 2.1 & 2.7 \\
\hline
\end{tabular}

Table 3. Journal of the high-resolution spectroscopic observations with the Asiago, SAAO, ESO, TNG and Rozhen telescopes (available only in electronic form at the CDS).

Table 4. Results of the multi-Gaussian fitting to the NaI and KI interstellar lines in the high resolution spectra of V838 Mon listed in Table 1 (available only in electronic form at the CDS).

Analysis of the high-resolution HST polarimetry images of the light-echo led Bond et al. (2003) to place a lower limit of $6 \mathrm{kpc}$ to the distance of V838 Mon. Working on the same HST material, Tylenda (2004) revised the distance to $8 \pm 2 \mathrm{kpc}$.

The spectro-photometric parallax to the B3V companion derived in Sect. 5 is $10 \mathrm{kpc}$. Furthermore, reversing the 
Table 5. Mean values and standard deviations of the radial velocity $(R V)$, full width at half maximum $(\Gamma)$ and equivalent width $(E W)$ of the NaI D and KI interstellar lines in the spectra of V838 Mon detailed in Table 2.

\begin{tabular}{|c|c|c|c|c|c|c|}
\hline & $\begin{array}{l}\mathrm{RV} \\
(\mathrm{km} \mathrm{sec}\end{array}$ & $\begin{array}{c}\sigma_{\mathrm{RV}} \\
-1)\end{array}$ & $\begin{array}{c}\Gamma \\
(\mathrm{km} \mathrm{s}\end{array}$ & $\begin{array}{c}\sigma_{\Gamma} \\
\left.c^{-1}\right)\end{array}$ & $\begin{array}{c}\text { E.W. } \\
(\AA)\end{array}$ & $\begin{array}{c}\sigma_{\mathrm{E} . \mathrm{W}} . \\
(\AA)\end{array}$ \\
\hline & \multicolumn{6}{|c|}{ blue component } \\
\hline NaI D1 & +38.4 & 1.8 & 29.8 & 1.7 & 0.610 & 0.035 \\
\hline NaI D2 & +36.4 & 1.8 & 29.0 & 1.1 & 0.625 & 0.044 \\
\hline \multirow[t]{2}{*}{ KI } & +38.3 & 2.1 & 18.1 & 3.4 & 0.100 & 0.054 \\
\hline & \multicolumn{6}{|c|}{ red component } \\
\hline NaI D1 & +65.7 & 1.8 & 18.3 & 1.7 & 0.329 & 0.038 \\
\hline NaI D2 & +64.3 & 1.5 & 19.4 & 0.9 & 0.366 & 0.009 \\
\hline KI & +65.4 & 1.8 & 13.4 & 3.4 & 0.128 & 0.017 \\
\hline
\end{tabular}

argument of the previous section, we can enter Table 2 or Fig. 5 with the reddening $E_{B-V}=0.87$ (corresponding to $A_{V}=2.7$ ) determined from the interstellar lines and the colors of the B3V companion, obtaining the same distance of $10 \mathrm{kpc}$ to V838 Mon.

Two other arguments are in favor of a large distance to V838 Mon, given its proximity to the galactic plane $(b=$ $-3^{\circ} 51^{\prime}$ galactic latitude). First, we have seen above that the HI radio observations in the direction of V838 Mon reveal three components whose velocity match exactly that of the three components seen in the interstellar absorption lines. Given the fact that the radio observations integrate along the whole line of sight through the Galaxy and that no $\mathrm{HI}$ is seen beyond V838 Mon (in the sense that no corresponding interstellar lines are detected in the high-resolution spectra), it is straightforward to conclude that V838 Mon lies at great distance with most of the galactic gas, in that direction, in front of it. Second, Schlegel et al. (1998) extinction maps are based on the amount of infrared emission by the dust integrated along the whole line of sight through the Galaxy. The amount of extinction affecting V838 Mon ( $E_{B-V}=0.87$ corresponding to $\left.A_{V}=2.7 \mathrm{mag}\right)$ match the value of Schegel et al. maps in that direction, supporting again the notion that V838 Mon lies at large galactocentric distances.

All these independent determinations support the conclusion that V838 Mon lies in the outer part of the disk of the Galaxy, at a distance of $\sim 10 \mathrm{kpc}$ from the Sun, corresponding to a galacto-centric distance of $\sim 17.5 \mathrm{kpc}$ and a height above the galactic plane of $\sim 650 \mathrm{pc}$.

Tylenda (2004) labeled as "naive interpretation" the early distance estimates of Munari et al. (2002a) and Kimeswenger et al. (2002) based on the first determinations of the angular expansion rate of the light-echo on early ground-based discovery images. The Munari et al. (2002a) implicit (but quite obvious) assumption was that the light-echo was originating in a circumstellar disk seen pole-on. Such an assumption was based on the fact that the NaI and KI lines were not tracing a circumstellar component, something to be expected in the case of a homogeneous spherical distribution of material centered on the object itself. It was only much later that high resolution HST imaging revealed that there is a clear void of circumstellar material

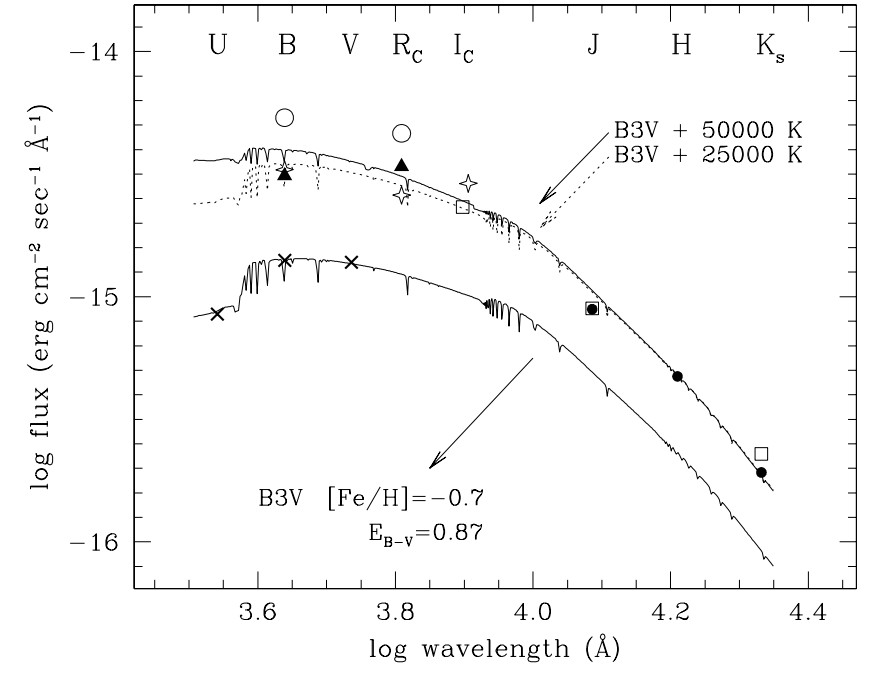

Fig. 10. The crosses represent the energy distribution of V838 Mon at the time of naked visibility of the hot companion $(V=16.05, B-V=$ $+0.68, U-B=-0.06)$ compared with a Kurucz's spectrum for a metal poor B3V star $\left(T_{\text {eff }}=19000 \mathrm{~K}, \lg g=4.0,[\mathrm{Fe} / \mathrm{H}]=-0.7\right)$ reddened by $E_{B-V}=+0.87$. The other symbols give the energy distribution of V838 Mon in quiescence: stars from Goranskii et al. (2004), triangles from Kimeswenger et al. (2002), squares from DENIS survey, dots from 2MASS survey, circles from USNO- $B$ magnitudes re-calibrated against the comparison sequence of Munari et al. (2002), giving $B=$ 15.28 and $R_{\mathrm{C}}=14.22$. The quiescence energy distribution is fitted with the combination of Kurucz's spectra for the B3V stars and the hotter companion.

precisely along the line of sight to V838 Mon, regardless of the true 3D shape of the circumstellar dust giving rise to the light-echo. So, the now "naive" approach was reasonable at the time.

\subsection{Progenitor}

Figure 10 shows the energy distribution of the B3 V companion in the $U B V$ bands $(\langle V\rangle=16.052,\langle B\rangle=16.736,\langle U\rangle=16.676$ average values from USNO $1.0 \mathrm{~m}$ photometry described in Sect. 1) compared with a Kurucz's synthetic spectrum (from the library of Munari et al. 2005) with parameters appropriate for the B3 V companion $\left(T_{\text {eff }}=19000 \mathrm{~K}, \log g=4.0\right.$, $[\mathrm{Fe} / \mathrm{H}]=-0.7)$. The match is excellent. In the same figure, the pre-outburst brightness of V838 Mon from various sources are plotted. In the infrared they come from the 2MASS and DENIS surveys, while the $B$ and $R_{\mathrm{C}}$ values are estimates of the same POSS-I and SERC plates according to different authors that used different calibrations. The entries at $I$ band (note the slightly different effective wavelengths) come from the DENIS and POSS-II surveys.

The figure clearly shows that the progenitor of the outbursting component was brighter and hotter than the $B 3 \mathrm{~V}$ companion. By exactly how much it depends on the still uncertain magnitude of V838 Mon on pre-outburst photographic plates. While the brightness seems well established in the infrared by the excellent agreement of DENIS and 2MASS data, it presents a large scatter in the estimates by different authors of the same 


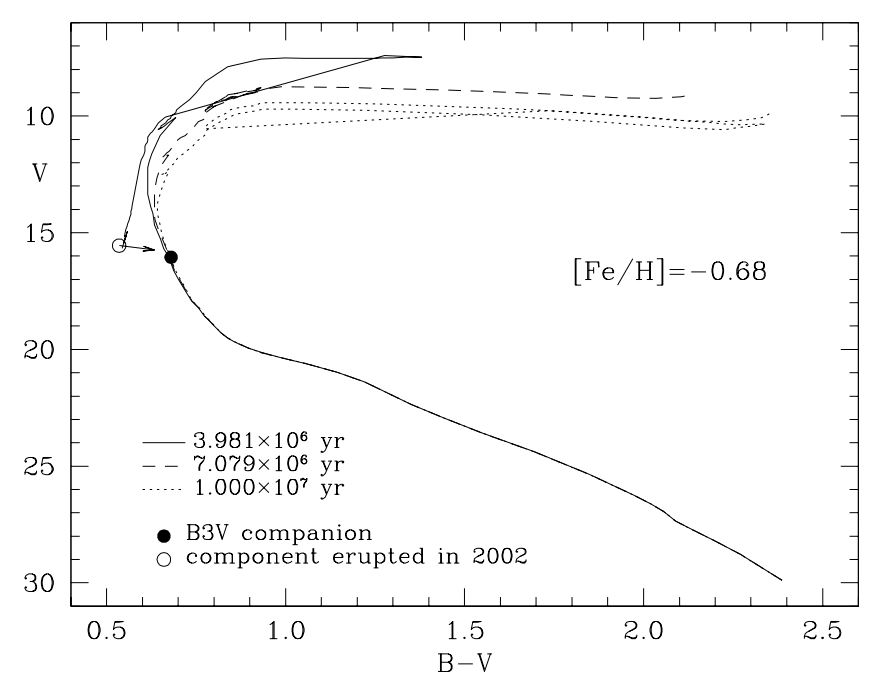

Fig. 11. Padova isochrones (from Bressan et al. 1993; Fagotto et al. 1994; Girardi et al. 2000) for $Z=0.004$ and three ages scaled to the distance $(10 \mathrm{kpc})$ and reddening $\left(E_{B-V}=0.87\right)$ of V838 Mon. The isochrones include the effect of mass loss and are corrected for the reddening-induced distortion described in Fiorucci \& Munari (2003, their Fig. 8). The open circle indicates the position of the V838 Mon component that erupted in 2002 if its temperature in quiescence was $50000 \mathrm{~K}$, and the arrow gives the shift if the temperature was $25000 \mathrm{~K}$ (cf. the energy distribution fit in Fig. 10).

POSS and SERC plates. It is clear from Fig. 10 that a careful re-evaluation of all existing pre-outburst photographic material would be beneficial in establishing the nature of V838 Mon prior to the outburst. Such a detailed analysis must first provide a perfect comparison sequence, a tight control over the plate measurement and its modeling, a careful knowledge of the exact band-pass profile of the given emulsion-filter-telescopeatmosphere combination and plate developing conditions, as well as careful application of color equation corrections.

The best fit to the V838 Mon quiescence data in Fig. 10 is the $\mathrm{B} 3 \mathrm{~V}$ plus a $50000 \mathrm{~K}$ star with $V=15.55$ and $B-V=$ +0.535 . Both stars are equally reddened by $E_{B-V}=0.87$. The combined spectrum of the two stars is plotted in Fig. 10 as a solid line. Ignoring our re-estimate of the magnitude of V838 Mon on the POSS and SERC plates listed in the caption of Fig. 10, a somewhat lower temperature for the eruption progenitor can be obtained, with a lower limit of $25000 \mathrm{~K}$. The combination of the spectra of the B3 V plus a $25000 \mathrm{~K}$ star with $V=15.74$ and $B-V=+0.634$ is plotted as a dotted line in Fig. 10.

The location of the components of the binary system V838 Mon is compared with theoretical isochrones in Fig. 11, for the $[\mathrm{Fe} / \mathrm{H}]=-0.7$ metallicity appropriate to the galactocentric distance of V838 Mon. The Padova theoretical isochrones have been scaled to a distance of $10 \mathrm{kpc}$ and $E_{B-V}=0.87$ reddening following the standard $R_{V}=3.1$ extinction law. The reddening dependent deformation over the HR plane discussed and quantified by Fiorucci \& Munari (2003, cf. their Fig. 8) for the $U B V R_{\mathrm{C}} I_{\mathrm{C}}$ photometric system (Vilnius and USA reconstructions) has been applied to the isochrones in Fig. 11.
As displayed by Fig. 11, there is a perfect match of the $[\mathrm{Fe} / \mathrm{H}]=-0.7$ isochrones with the observed magnitude and colors of the $\mathrm{B} 3 \mathrm{~V}$ component, with the progenitor of the outbursting companion lying on the isochrone for an age of 4 million yr, close to the position of the central ignition of carbon for an initial mass of $\sim 65 M_{\odot}$. The progenitor lies close to the region where Wolf-Rayet stars are usually located. It would be harder to fit with theoretical evolutionary tracks the position of the progenitor of the outbursting component if its temperature would be decreased to the lower limit of $25000 \mathrm{~K}$ of Fig. 10, indicated by the head of the arrow in Fig. 11.

We therefore conclude that the progenitor of the outbursting component of the binary system V838 Mon was hotter, brighter and born far more massive than the $7 M_{\odot}$ B3 V companion, of an age not far from 4 million yr. In this respect, the circumstellar scattering material giving rise to the light-echo is probably the result of the massive mass loss that such massive objects experience, where $\sim$ half of the mass is lost during the main sequence phase. Given the location of V838 Mon in the outskirts of the Galactic disk at galactic longitude $l=218^{\circ}$, the large mass and young age could sound problematic. However, young clusters and massive stars at great distances in the anti-center direction are already known. For ex., Fitzgerald \& Moffat (1976) reported a distance of $\sim 7 \mathrm{kpc}$ for the very young cluster Ruprecht $44\left(l=245^{\circ}\right)$ rich in O and WR stars, or Marco et al. (2001) derived a distance of $6 \mathrm{kpc}$ and an age of $4 \mathrm{Myr}$ for the cluster NGC $1893\left(l=173^{\circ}\right)$ that harbors O5V member stars (which mass is $\sim 65 M_{\odot}$ according to Straižys \& Kuriliené (1981) tabulation).

The outburst experienced in 2002 does not appear as the terminal event in the life of the massive progenitor, but instead more probably as a thermonuclear shell flash in the outer layers of the star as could be expected in the case of He after most of the H-rich outer envelope has been blown away by the strong wind that characterize this type of stars. A detailed analysis of the evolutionary status of the progenitor will be presented elsewhere (Munari et al. in preparation).

Acknowledgements. This work has been supported in part by Italian COFIN-2002 grant, Polish KBN Grant No. 2 P03D 019 25, and NSF and NASA grants to ASU. We would like to thank F. d'Antona and O. Straniero for useful comments.

\section{References}

Banerjee, D. P. K., \& Ashok, N. M. 2002, A\&A, 395, 161

Barsukova, E. A., Borisov, N. V., Goranskii, V. P., et al. 2002, in Classical Nova Explosions, ed. M. Hernanz, \& J. José, Am. Inst. Phys. Conf. Proc. 637, 303

Bertelli, G., Bressan, A., Chiosi, C., Ng, Y. K., \& Ortolani, S. 1995, A\&A, 301, 381

Bessell, M. S. 2000, PASP, 112, 961

Bond, H. E., Henden, A., Levay, Z. G., et al. 2003, Nature, 422, 405

Boschi, F., \& Munari, U. 2004, A\&A, 418, 869

Brand, J., \& Blitz, L. 1993, A\&A, 275, 67

Bressan, A., Fagotto, F., Bertelli, G., et al. 1993, A\&AS, 100, 647

Brown, N. J. 2002, IAU Circ., 7785

Crause, L., Lawson, W. A., Kilkenny, D., et al. 2003, MNRAS, 341, 785

Desidera, S., \& Munari, U. 2002, IAUC, 7982 
Desidera, S., Giro, E., Munari, U., et al. 2004, A\&A, 414, 591

Dias, W. S., Alessi, B. S., Moitinho, A., \& Lépine, J. R. D. 2002, A\&A, 389, 871

Drimmel, R., Cabrera-Lavers, A., \& López-Corredoira, M. 2003, A\&A, 409, 205

Evans, A., Geballe, T. R., Rushton, M. T., et al. 2003, MNRAS, 343, 1054

Fagotto, F., Bressan, A., Bertelli, G., \& Chiosi, C. 1994, A\&AS, 105, 29

Fiorucci, M., \& Munari, U. 2003, A\&A, 401, 781

Fitzgerald, M. P. 1970, A\&A, 4, 234

Fitzgerald, M. P., \& Moffat, A. F. J. 1976, A\&A, 50, 149

Friel, E. D., Janes, K. A., Tavarez, M., et al. 2002, AJ, 124, 2693

Girardi, L., Bressan, A., Bertelli, G., \& Chiosi, C. 2000, A\&AS, 141, 371

Goranskii, V. P., Kusakin, A. V., Metlova, N. V., et al. 2002, Astron. Lett., 28, 691

Goranskij, V. P., Shugarov, S. Yu., Barsukova, E. A., \& Kroll, P. 2004, IBVS 5511

Hartmann, L., \& Burton, W. B. 1997, Atlas of Galactic Neutral Hydrogen (Cambridge Univ. Press)

Henden, A., Munari, U., \& Schwartz, M. 2002, IAUC, 7859

Høg, E., Fabricius, C., Makarov, V. V., et al. 2000, A\&A, 355, L27

Hou, J.-L., Chang, R.-X., \& Chen, L. 2002, ChJAA, 2, 17

Houk, N. 2004, home page of the Michigan Project: http://www .astro.1sa.umich.edu/users/hdproj/

Houk, N., \& Swift, C. 1999, Michigan catalogue of two-dimensional spectral types for the HD Stars, 5, Department of Astronomy, University of Michigan

Hron, J. 1987, A\&A, 176, 34

Kato, T. 2003, A\&A, 399, 695

Kimeswenger, S., Lederle, C., Schmeja, S., \& Armsdorfer, B. 2002, MNRAS, 336, L43

Kipper, T., Klochkova, V. G., Annuk, K., et al. 2004, A\&A, 416, 1107

Kolev, D., Mikolajewski, M., Tomov, T., et al. 2002, Collected Papers. Physics (Shumen, Bulgaria: Shumen University Press), 147
Landolt, A. U. 1983, AJ, 88, 439

Landolt, A. U. 1992, AJ, 104, 304

Lynga, G. 1987, Catalogue of Open Cluster Data. Lund Observatory, Sweden

Marco, A., Bernabeu, G., \& Negueruela, I. 2001, AJ, 121, 2075

Mendez, R. A., \& van Altena, W. F. 1998, A\&A, 330, 910

Munari, U., \& Zwitter, T. 1997, A\&A, 318, 269

Munari, U., Henden, A., Kiyota, S., et al. 2002a, A\&A, 389, L51

Munari, U., Desidera, S., \& Henden, A. 2002b, IAUC, 8005

Munari, U., Henden, A., Corradi, R. M. L., \& Zwitter, T. 2002c, in Classical Nova Explosions, ed. M. Hernanz, \& J. José, Am. Inst. Phys. Conf. Proc., 637, 52

Munari, U., Sordo, R., Castelli, F., \& Zwitter, T. 2005, A\&A, submitted [arXiv:astro-ph/0502047]

Neckel, Th., \& Klare, G. 1980, A\&AS, 42, 251

Osiwala, J. P., Mikolajewski, M., Tomov, T., et al. 2003, in Symbiotic Stars Probing Stellar Evolution, ed. L. Corradi et al., ASP Conf. Ser., 303, 240

Orio, M., Starrfield, S. G., \& Tepedenlegliolu, E. 2004, IAUC, 8110

Retter, A., \& Marom, A. 2003, MNRAS, 345, L25

Rushton, M. T., Coulson, I. M., Evans, A., et al. 2003, A\&A, 412, 767

Schlegel, D. J., Finkbeiner, D. P., \& Davis, M. 1998, ApJ, 500, 525

Soker, N., \& Tylenda, R. 2003, ApJ, 582, L105

Straižys, V., \& Kuriliené, G. 1981, ApSpSc, 80, 353

Sugerman, B. E. K. 2003, AJ, 126, 1939

Tylenda, R. 2004, A\&A, 414, 223

Vallenari, A., Bertelli, G., \& Schmidtobreick, L. 2000, A\&A, 361, 73

Vallenari, A., Pasetto, S., Bertelli, G., Chiosi, C., \& Spagna, A. 2004 A\&A, submitted

Wagner, R. M., \& Starrfield, S. G. 2002, IAUC, 7992

Wagner, R. M., Schwarz, G., Starrfield, S., \& Szkody, P. 2003, IAUC, 8202

Wisniewski, J. P., Morrison, N. D., Bjorkman, K. S., et al. 2003a, ApJ, 588, 486

Wisniewski, J. P., Bjorkman, K. S., \& Magalhaes, M. 2003b, ApJ, 598, L43 